\title{
CURVATURES OF CONFLICT SURFACES IN EUCLIDEAN 3-SPACE
}

\author{
JORGE SOTOMAYOR \\ Instituto de Matemática e Estatística \\ Universidade de São Paulo \\ Caixa Postal 66281, São Paulo, SP, Cep 05315-970, Brazil \\ E-mail: sotp@ime.usp.br \\ DIRK SIERSMA \\ Mathematisch Instituut \\ Universiteit Utrecht \\ Postbus 80.010, 3508 TA Utrecht, The Netherlands \\ E-mail:siersma@math.uu.nl \\ RONALDO GARCIA \\ Instituto de Matemática e Estatística \\ Universidade Federal de Goiás \\ Caixa Postal 131, Goiânia, GO, Cep 74001-970, Brazil \\ E-mail: ragarcia@mat.ufg.br
}

\begin{abstract}
This article extends to three dimensions results on the curvature of the conflict curve for pairs of convex sets of the plane, established by Siersma [3]. In the present case a conflict surface arises, equidistant from the given convex sets. The Gaussian, mean curvatures and the location of umbilic points on the conflict surface are determined here. Initial results on the Darbouxian type of umbilic points on conflict surfaces are also established. The results are expressed in terms of the principal directions and on the curvatures of the borders of the given convex sets.
\end{abstract}

1. Introduction. Let $A_{1}$ and $A_{2}$ be closed non-empty sets in Euclidean space $R^{3}$, which is endowed with the standard orientation and the distance

$$
d(p, q)=|p-q|=\langle p-q, p-q\rangle^{1 / 2},
$$

where $\langle$,$\rangle is the standard Euclidean inner product in R^{3}$.

1991 Mathematics Subject Classification: Primary 53A04; Secondary 53C03.

This work was done under the project PRONEX/FINEP/MCT — Conv. 76.97.1080.00 Teoria Qualitativa das Eq. Dif. Ordinárias.

The paper is in final form and no version of it will be published elsewhere. 
The conflict set $C\left(A_{1}, A_{2}\right)$ between $A_{1}$ and $A_{2}$ is defined by

$$
C\left(A_{1}, A_{2}\right)=\left\{p ; d\left(p, A_{1}\right)=d\left(p, A_{2}\right)\right\},
$$

where $d(p, A)=\inf \{d(p, q) ; q \in A\}$. The set $C\left(A_{1}, A_{2}\right)$ is also viewed as the common boundary between the territory of $A_{1}$ relative to $A_{2}$, defined by

$$
\operatorname{Terr}\left(A_{1}, A_{2}\right)=\left\{p ; d\left(p, A_{1}\right)<d\left(p, A_{2}\right)\right\},
$$

and $\operatorname{Terr}\left(A_{2}, A_{1}\right)$, which is the territory of $A_{2}$ relative to $A_{1}$.

The set $C\left(A_{1}, A_{2}\right)$ is also called the bisector or the equidistant set between $A_{1}$ and $A_{2}[7]$.

In this paper we consider only the case where $A_{i}$ are convex closed sets, with disjoint interiors and smooth regular boundaries $B_{i}=\partial A_{i}$ of class $C^{k}, k \geq 2$. Assume that the surfaces $B_{i}$ are oriented by their unit normal vector fields $N_{i}$, pointing towards the interior of $A_{i}$.

To each point $p$ on the closed set $E_{i}=R^{3} \backslash \operatorname{Int}\left(A_{i}\right)$, we associate its projection $\Pi_{i}(p)=p_{i}$ onto $B_{i}$, characterized by

$$
d\left(p, A_{i}\right)=\left\langle\Pi_{i}(p)-p, N_{i}\left(\Pi_{i}(p)\right)\right\rangle .
$$

From the tubular neighborhood properties and the convexity hypothesis, it follows that $\Pi_{i}$ is a retraction of class $C^{r-1}$ of a neighborhood of $E_{i}$ onto $B_{i}$; see [6], Vol. 1 . Therefore $C=C\left(A_{1}, A_{2}\right)$, is defined implicitly by the zero level set of the function

$$
c(p)=d\left(p, A_{2}\right)-d\left(p, A_{1}\right),
$$

which is regular of class $C^{r-1}$ and satisfies

$$
\nabla c(p)=N_{1}\left(\Pi_{1}(p)\right)-N_{2}\left(\Pi_{2}(p)\right) .
$$

It is clear that this regularity property fails if the convexity and disjointness hypotheses are not imposed.

In this paper the conflict surface $C$ will be oriented by the unit normal $N$, along $\nabla c$, i.e. pointing from $A_{2}$ towards $A_{1}$ :

$$
N=\left|N_{1}\left(\Pi_{1}(p)\right)-N_{2}\left(\Pi_{2}(p)\right)\right|^{-1}\left[N_{1}\left(\Pi_{1}(p)\right)-N_{2}\left(\Pi_{2}(p)\right)\right] .
$$

To simplify the notation write $\nu(V)=|V|^{-1} V$ for the normalization of a nonvanishing vector $V$. Therefore,

$$
N=\nu\left(N_{1}\left(\Pi_{1}(p)\right)-N_{2}\left(\Pi_{2}(p)\right)\right) .
$$

A positive moving frame attached to $C$ is therefore given by $\left\{T_{1}, T_{2}, N\right\}$, where:

$$
\begin{aligned}
& T_{1}=\nu\left(N_{1}\left(\Pi_{1}(p)\right)+N_{2}\left(\Pi_{2}(p)\right)\right), \\
& T_{2}=\nu\left(N_{1}\left(\Pi_{1}(p)\right) \wedge N_{2}\left(\Pi_{2}(p)\right)\right) .
\end{aligned}
$$

Notice that the vector fields $T_{i}$ are singular at points $p$ of the closed set $M=$ $M\left(A_{1}, A_{2}\right)$, where $N_{1}\left(\Pi_{1}(p)\right)+N_{2}\left(\Pi_{2}(p)\right)=0$, which occurs when the distance from $C$ to $A_{i}$,

$$
r(p)=d\left(p, A_{1}\right)=d\left(p, A_{2}\right),
$$

is minimal, assuming the value $d_{m}=\frac{1}{2} d\left(A_{1}, A_{2}\right)$. Under the condition of strict convexity of $B_{i}$ at points of the sets $\Pi_{i}(M), M$ reduces to a unique point $p_{m}$. 
Recall that strict convexity of $B_{i}$ at $p_{i}$ means that $D N_{i}$ is an automorphism of the tangent space $T B_{i}$, with the usual identification of the tangent space $T B_{i}$ at $p_{i}$ with that of the unit sphere $T S^{2}$, at $N_{i}\left(p_{i}\right)$. In terms of the principal curvatures $k_{1}^{i} \leq k_{2}^{i}$, which are the eigenvalues of $-D N_{i}$, the condition of strict convexity (resp. convexity) means that both are positive (resp. non-negative). In terms of the Gaussian curvature

$$
\mathcal{K}^{i}=k_{1}^{i} \cdot k_{2}^{i}=\operatorname{det}\left(-D N_{i}\right),
$$

this means that $\mathcal{K}^{i}>0$ (resp. $\mathcal{K}^{i} \geq 0$ ). Recall also that the mean curvature of $B_{i}$ is given by

$$
\mathcal{H}^{i}=\frac{1}{2} \operatorname{trace}\left(-D N_{i}\right)=\frac{1}{2}\left(k_{1}^{i}+k_{2}^{i}\right)
$$

The expression

$$
\mathcal{U}^{i}=\frac{1}{2}\left(k_{2}^{i}-k_{1}^{i}\right)=\sqrt{\left(\mathcal{H}^{i}\right)^{2}-\mathcal{K}^{i}},
$$

also called skew curvature, whose zeros locate the umbilic points of the surface, will be also of later of use here.

Theorem 1 of this paper establishes expressions for the Gaussian and mean curvatures of the surface $C=C\left(A_{1}, A_{2}\right)$. These results can be regarded as the starting step for the investigation of the principal configuration of $C$ [4]. Recall that this configuration is defined by the principal curvatures, the umbilic points, the fields of principal directions (which are the eigenspaces of $-D N_{i}$ ), and their integral curves (including the periodic principal lines). The location of umbilic points and the slope of principal directions on $C \backslash M$ is established in Corollary 3. Initial results on the Darbouxian type of the umbilic points on $M=M\left(A_{1}, A_{2}\right)$ are established in Section 3.

A complete study of the dependence on $B_{i}$ of the principal configuration on conflict surfaces will not be carried out in this work. However, the curvature formulas of this paper are expressed in terms of some elements of the principal configurations of the surfaces $B_{i}$.

Global topological properties of conflict sets, such as their connectivity, have been studied in [7]. The basic smoothness and curvature expressions of the conflict curve for convex sets in the plane have been established in [3], where interesting connections with Euclidean geometry of conics can also be found.

Acknowledgements. This work was prepared with the partial support from $\mathrm{CNPq}$ FINEP and FUNAPE.

2. Curvatures of conflict surfaces. Let $p \in C \backslash M$ be such that $p_{i}=\Pi_{i}(p)$ are non-umbilic points in $B_{i}$. Let $\left\{E_{1}^{i}, E_{2}^{i}, N_{i}\right\}$ be positive principal frames on $B_{i}$ around $p_{i}$. This means that

$$
D N_{i} \cdot E_{j}^{i}=-k_{j}^{i} E_{j}^{i},
$$

where $k_{1}^{i}<k_{2}^{i}, i=1,2$, are the principal curvatures of $B_{i}$. Denote by $\alpha_{i}$ the angle between the vectors $E_{1}^{i}$ and $F_{1}^{i}=\nu\left(D \Pi_{i}(p) T_{1}\right)$. Write

$$
\tau_{g}^{i}=\tau_{g}^{i}\left(\alpha_{i}\right)=\left(k_{2}^{i}-k_{1}^{i}\right) \sin \alpha_{i} \cos \alpha_{i}
$$

for the geodesic torsion along the unit vector $F_{1}^{i}$ on $B_{i}$. 
Write

$$
k_{n}^{i}=k_{n}^{i}\left(\alpha_{i}\right)=k_{1}^{i} \cos ^{2}\left(\alpha_{i}\right)+k_{2}^{i} \sin ^{2}\left(\alpha_{i}\right),
$$

which, by Euler's formula, is the normal curvature of $B_{i}$ in the direction of $F_{1}^{i}=$ $\nu\left(D \Pi_{i}(p) T_{1}\right)$.

Similarly, the normal curvature of $B_{i}$ in the direction of $F_{2}^{i}=\nu\left(D \Pi_{i}(p) T_{2}\right)$ is given by

$$
k_{n \perp}^{i}=k_{n \perp}^{i}\left(\alpha_{i}\right)=k_{n}^{i}\left(\alpha_{i}+\frac{\pi}{2}\right)=k_{2}^{i} \sin ^{2}\left(\alpha_{i}\right)+k_{1}^{i} \cos ^{2}\left(\alpha_{i}\right) .
$$

Denote by $A_{i}^{r}$ the convex set of points at distance $r \geq 0$ from $A_{i}$; its border is the surface $B_{i}^{r}$ obtained by moving each point $p_{i}$ on $B_{i}$ to $\Sigma^{r}\left(p_{i}\right)=p_{i}-r N_{i}\left(p_{i}\right)$. By restriction, $\Pi_{i}$ defines the diffeomorphism $\left(\Sigma^{r}\right)^{-1}$ of $B_{i}^{r}$ onto $B_{i}$ and, by composition, it defines the retraction $\Pi_{i}^{r}=\Pi_{i} \circ\left(\Sigma^{r}\right)^{-1}$ onto $B_{i}^{r}$.

The principal frames $\left\{E_{1}^{i}, E_{2}^{i}, N_{i}\right\}$ on $B_{i}$ are parallel translated along the normals to principal frames on $B_{i}^{r}$. This follows from the fact that $\Sigma^{r}$ preserves the principal direction fields as well as the umbilic points. The principal curvatures however change into $k_{j}^{i}(r)=\frac{k_{j}^{i}}{1+r k_{j}^{i}}$, [6], Vol. 3.

The above expressions for $\tau_{g}^{i}, k_{n}^{i}$ and $k_{n \perp}^{i}$ on $B_{i}$, being defined in terms of principal curvatures, can be obviously modified to be valid on $B_{i}^{r}$ and denoted respectively by $\tau_{g}^{i}(r), k_{n}^{i}(r)$ and $k_{n \perp}^{i}(r)$. For instance:

$$
\begin{aligned}
\tau_{g}^{i}(r) & =\tau_{g}^{i}\left(r, \alpha_{i}\right)=\left(k_{2}^{i}(r)-k_{1}^{i}(r)\right) \sin \alpha_{i} \cos \alpha_{i}, \\
k_{n}^{i}(r) & =k_{1}^{i}(r) \cos ^{2}\left(\alpha_{i}\right)+k_{2}^{i}(r) \sin ^{2}\left(\alpha_{i}\right), \\
k_{n \perp}^{i}(r) & =k_{2}^{i}(r) \sin ^{2}\left(\alpha_{i}\right)+k_{1}^{i}(r) \cos ^{2}\left(\alpha_{i}\right) .
\end{aligned}
$$

Denote by $\phi$ the angle between $N_{i}$ and $T_{1}$. For future reference, notice that

$$
\sin \phi=\frac{1}{2}\left|N_{1}\left(\Pi_{1}(p)\right)-N_{2}\left(\Pi_{2}(p)\right)\right|=\left\langle F_{1}^{1}, T_{1}\right\rangle=\left\langle F_{1}^{2}, T_{1}\right\rangle .
$$

Theorem 1. Let $\mathcal{K}^{i}=\mathcal{K}^{i}(r)=k_{1}^{i}(r) k_{2}^{i}(r), \mathcal{H}^{i}=\mathcal{H}^{i}(r)=\frac{1}{2}\left(k_{1}^{i}(r)+k_{2}^{i}(r)\right)$ and $\mathcal{U}^{i}=\mathcal{U}^{i}(r)=\frac{1}{2}\left(k_{2}^{i}(r)-k_{1}^{i}(r)\right)$, where $k_{j}^{i}(r)=\frac{k_{j}^{i}}{1+r k_{j}^{i}}$.

a) The Gaussian curvature of the conflict surface $C$ is given by

$$
\mathcal{K}=\frac{1}{2}\left[\frac{1}{2}\left(\mathcal{K}^{1}+\mathcal{K}^{2}\right)-\mathcal{H}^{1} \mathcal{H}^{2}+\cos \left(2\left(\alpha_{2}-\alpha_{1}\right)\right) \mathcal{U}^{1} \mathcal{U}^{2}\right] .
$$

b) The mean curvature of the conflict surface $C$ is given by

$$
\mathcal{H}=\left(\frac{1+\sin ^{2} \phi}{4 \sin ^{2} \phi}\right)\left[\left(\mathcal{H}^{1}-\mathcal{H}^{2}\right)+\left(\frac{\cos ^{2} \phi}{1+\sin ^{2} \phi}\right)\left(\cos \left(2 \alpha_{1}\right) \mathcal{U}^{1}-\cos \left(2 \alpha_{2}\right) \mathcal{U}^{2}\right)\right] .
$$

The proof of this theorem will follow from the next proposition.

Proposition 2. With the above notation, at points of $C \backslash M$

$$
\begin{aligned}
& D N . T_{1}=\frac{\sin \phi}{2}\left[k_{n}^{2}(r)-k_{n}^{1}(r)\right] T_{1}+\frac{1}{2}\left[\tau_{g}^{2}(r)-\tau_{g}^{1}(r)\right] T_{2}, \\
& D N . T_{2}=\frac{1}{2}\left[\tau_{g}^{2}(r)-\tau_{g}^{1}(r)\right] T_{1}+\frac{1}{2 \sin \phi}\left[k_{n \perp}^{2}(r)-k_{n \perp}^{1}(r)\right] T_{2} .
\end{aligned}
$$


Proof. The conclusion follows from the calculation of the inner products in

$$
\begin{aligned}
& D N\left(T_{1}\right)=\left\langle D N\left(T_{1}\right), T_{1}\right\rangle T_{1}+\left\langle D N\left(T_{1}\right), T_{2}\right\rangle T_{2}, \\
& D N\left(T_{2}\right)=\left\langle D N\left(T_{2}\right), T_{1}\right\rangle T_{1}+\left\langle D N\left(T_{2}\right), T_{2}\right\rangle T_{2} .
\end{aligned}
$$

Differentiation of $N$ gives:

$$
D N=\frac{D N_{1} \cdot D \Pi_{1}^{r}-D N_{2} \cdot D \Pi_{2}^{r}}{2 \sin \phi}+2 D\left[(\sin \phi)^{-1}\right] \cdot\left[N_{1}\left(\Pi_{1}^{r}(p)\right)-N_{2}\left(\Pi_{2}^{r}(p)\right)\right] .
$$

This shows that for the present analysis the contribution of the second term is null.

The basis $\left\{T_{1}, T_{2}\right\}$ on $C$ projects along $\Pi_{i}^{r}$ onto the orthonormal bases $\left\{F_{1}^{i}, F_{2}^{i}\right\}$ on $B_{i}^{r}$.

Calculation shows that

$$
\begin{aligned}
& \left\{F_{1}^{1}=v\left(N_{2}-\left\langle N_{1}, N_{2}\right\rangle N_{1}\right), F_{2}^{1}=T_{2}\right\} \\
& \left\{F_{1}^{2}=v\left(-N_{1}+\left\langle N_{1}, N_{2}\right\rangle N_{2}\right), F_{2}^{2}=T_{2}\right\} .
\end{aligned}
$$

Therefore,

$$
D \Pi_{i}^{r}\left(T_{1}\right)=\sin \phi F_{1}^{i} \quad \text { and } \quad D \Pi_{i}^{r}\left(T_{2}\right)=F_{2}^{i} .
$$

The bases $E^{i}$ and $F^{i}$ are related by

$$
\begin{aligned}
& F_{1}^{i}=\cos \left(\alpha_{i}\right) E_{1}^{i}+\sin \left(\alpha_{i}\right) E_{2}^{i}, \quad F_{2}^{i}=-\sin \left(\alpha_{i}\right) E_{1}^{i}+\cos \left(\alpha_{i}\right) E_{2}^{i}, \\
& D N_{i} \cdot D \Pi_{i}^{r}\left(T_{1}\right)=\sin \phi D N_{i}\left(\cos \left(\alpha_{i}\right) E_{1}^{i}+\sin \left(\alpha_{i}\right) E_{2}^{i}\right) \\
& =\sin \phi\left[\cos \left(\alpha_{i}\right) D N_{i}\left(E_{1}^{i}\right)+\sin \left(\alpha_{i}\right) D N_{i}\left(E_{2}^{i}\right)\right] \\
& =\sin \phi\left[-k_{1}^{i}(r) \cos \left(\alpha_{i}\right) E_{1}^{i}-k_{2}^{i}(r) \sin \left(\alpha_{i}\right) E_{2}^{i}\right] \\
& =\sin \phi\left\{-k_{1}^{i}(r) \cos \left(\alpha_{i}\right)\left[\cos \left(\alpha_{i}\right) F_{1}^{i}-\sin \left(\alpha_{i}\right) F_{2}^{i}\right]\right. \\
& \left.-k_{2}^{i}(r) \sin \left(\alpha_{i}\right)\left[\cos \left(\alpha_{i}\right) F_{1}^{i}+\sin \left(\alpha_{i}\right) F_{2}^{i}\right]\right\} \\
& =\sin \phi\left\{\left[-k_{1}^{i}(r) \cos ^{2}\left(\alpha_{i}\right)-k_{2}^{i}(r) \sin ^{2}\left(\alpha_{i}\right)\right] F_{1}^{i}\right. \\
& \left.+\left[\left(k_{1}^{i}(r)-k_{2}^{i}(r)\right) \sin \left(\alpha_{i}\right) \cos \left(\alpha_{i}\right)\right] F_{2}^{i}\right\} \\
& =\sin \phi\left\{\left[-k_{n}^{i}(r)\right] F_{1}^{i}+\left[-\tau_{g}^{i}(r)\right] F_{2}^{i}\right\} \text {. }
\end{aligned}
$$

Analogously,

$$
\begin{aligned}
& D N_{i} \cdot D \Pi_{i}^{r}\left(T_{2}\right)=D N_{i}\left(\cos \left(\alpha_{i}+\frac{\pi}{2}\right) E_{1}^{i}+\sin \left(\alpha_{i}+\frac{\pi}{2}\right) E_{2}^{i}\right) \\
& =\left[k_{1}^{i}(r) \sin \left(\alpha_{i}\right) E_{1}^{i}-k_{2}^{i}(r) \cos \left(\alpha_{i}\right) E_{2}^{i}\right] \\
& =\left\{k_{1}^{i}(r) \sin \left(\alpha_{i}\right)\left[\cos \left(\alpha_{i}\right) F_{1}^{i}-\sin \left(\alpha_{i}\right) F_{2}^{i}\right]\right. \\
& \left.-k_{2}^{i}(r) \cos \left(\alpha_{i}\right)\left[\sin \left(\alpha_{i}\right) F_{1}^{i}+\cos \left(\alpha_{i}\right) F_{2}^{i}\right]\right\} \\
& =\left\{k_{1}^{i}(r) \sin \left(\alpha_{i}\right) \cos \left(\alpha_{i}\right) F_{1}^{i}-k_{1}^{i}(r) \sin ^{2}\left(\alpha_{i}\right) F_{2}^{i}\right. \\
& \left.-k_{2}^{i}(r) \cos \left(\alpha_{i}\right) \sin \left(\alpha_{i}\right) F_{1}^{i}-k_{2}^{i}(r) \cos ^{2}\left(\alpha_{i}\right) F_{2}^{i}\right\} \\
& =\left\{\left[\left(k_{1}^{i}(r)-k_{2}^{i}(r)\right) \sin \left(\alpha_{i}\right) \cos \left(\alpha_{i}\right)\right] F_{1}^{i}\right. \\
& \left.-\left[k_{1}^{i}(r) \sin ^{2}\left(\alpha_{i}\right)+k_{2}^{i}(r) \cos ^{2}\left(\alpha_{i}\right)\right] F_{2}^{i}\right\} \\
& =\left\{\left[-\tau_{g}^{i}(r)\right] F_{1}^{i}-\left[k_{n \perp}^{i}(r)\right] F_{2}^{i}\right\} \text {. }
\end{aligned}
$$

Therefore,

$$
D N\left(T_{1}\right)=\frac{\sin \phi}{2 \sin \phi}\left\{\left[-k_{n}^{1}(r) F_{1}^{1}-\tau_{g}^{1}(r) F_{2}^{1}\right]-\left[-k_{n}^{2}(r) F_{1}^{2}-\tau_{g}^{2}(r) F_{2}^{2}\right]\right\}
$$




$$
\begin{aligned}
& =\frac{1}{2}\left\{\left[k_{n}^{2}(r) F_{1}^{2}-k_{n}^{1}(r) F_{1}^{1}\right]+\left[\tau_{g}^{2}(r)\right] F_{2}^{2}-\left[\tau_{g}^{1}(r) F_{2}^{1}\right]\right\}, \\
D N\left(T_{2}\right) & =\frac{1}{2 \sin \phi}\left\{\left[-\tau_{g}^{1}(r)\right] F_{1}^{1}-\left[k_{n \perp}^{1}(r)\right] F_{2}^{1}+\left[\tau_{g}^{2}(r)\right] F_{1}^{2}+\left[k_{n \perp}^{2}(r)\right] F_{2}^{2}\right\} \\
& =\frac{1}{2 \sin \phi}\left\{\left[k_{n \perp}^{2}(r) F_{2}^{2}-k_{n \perp}^{1}(r) F_{2}^{1}\right]+\left[\tau_{g}^{2}(r)\right] F_{1}^{2}-\left[\tau_{g}^{1}(r)\right] F_{1}^{1}\right\} .
\end{aligned}
$$

Performing the inner products, taking into account that

$$
\sin \phi=\cos \left(\frac{\pi}{2}-\phi\right)=\left\langle F_{1}^{1}, T_{1}\right\rangle=\left\langle F_{1}^{2}, T_{1}\right\rangle,
$$

finishes the proof.

Remark 1. Notice that the proof of the last proposition uses only the principal frames at points (and not on open sets) of $B^{i}$. Therefore the calculations for $D N$ hold also at points of $C \backslash M$ whose projections on either one (or both) of the surfaces $B^{i}$ are umbilic points, in that case the corresponding expressions $\tau_{g}^{i}(r)$ vanish. Proposition 4 will deal with a preliminary analysis of the case of points on $M$, where $T_{1}$ and the angles $\alpha_{i}$ are not defined.

Proof of Theorem 1.

a) The Gaussian curvature of $C$ is given by $\operatorname{det}(-D N)$. Therefore, taking into account that

$$
\begin{gathered}
k_{n}^{2}(r)=\mathcal{H}^{2}-\mathcal{U}^{2} \cos 2 \alpha_{2}, \quad k_{n}^{1}(r)=\mathcal{H}^{1}-\mathcal{U}^{1} \cos 2 \alpha_{1}, \quad k_{n \perp}^{2}(r)=\mathcal{H}^{2}+\mathcal{U}^{2} \cos 2 \alpha_{2}, \\
k_{n \perp}^{1}(r)=\mathcal{H}^{1}+\mathcal{U}^{1} \cos 2 \alpha_{1}, \quad \tau_{g}^{2}(r)-\tau_{g}^{1}(r)=\mathcal{U}^{2} \sin 2 \alpha_{2}-\mathcal{U}^{1} \sin 2 \alpha_{1} \\
\text { and }\left(\mathcal{U}^{i}\right)^{2}=\left(\mathcal{H}^{i}\right)^{2}-\mathcal{K}^{i},
\end{gathered}
$$

we obtain

$$
\begin{aligned}
\mathcal{K}= & \frac{1}{4}\left\{\left[k_{n}^{2}(r)-k_{n}^{1}(r)\right]\left[k_{n \perp}^{2}(r)-k_{n \perp}^{1}(r)\right]-\left[\tau_{g}^{2}(r)-\tau_{g}^{1}(r)\right]^{2}\right\} \\
= & \frac{1}{4}\left\{\left(\mathcal{H}^{2}-\mathcal{U}^{2} \cos 2 \alpha_{2}\right)-\left(\mathcal{H}^{1}-\mathcal{U}^{1} \cos 2 \alpha_{1}\right)\right]\left[\left(\mathcal{H}^{2}+\mathcal{U}^{2} \cos 2 \alpha_{2}\right)\right. \\
& \left.\left.-\left(\mathcal{H}^{1}+\mathcal{U}^{1} \cos 2 \alpha_{1}\right)\right]-\left[\mathcal{U}^{2} \sin 2 \alpha_{2}-\mathcal{U}^{1} \sin 2 \alpha_{1}\right]^{2}\right\} \\
= & \frac{1}{2}\left[\frac{1}{2}\left(\mathcal{K}^{1}+\mathcal{K}^{2}\right)-\mathcal{H}^{1} \mathcal{H}^{2}+\cos \left(2\left(\alpha_{2}-\alpha_{1}\right)\right) \mathcal{U}^{1} \mathcal{U}^{2}\right] .
\end{aligned}
$$

b) The mean curvature is given by trace $\left(-\frac{1}{2} D N\right)$.

From the relations

$$
\begin{gathered}
k_{n}^{2}(r)-k_{n}^{1}(r)=\mathcal{H}^{2}-\mathcal{H}^{1}+\mathcal{U}^{1} \cos 2 \alpha_{1}-\mathcal{U}^{2} \cos 2 \alpha_{2}, \\
\text { and } k_{n \perp}^{2}(r)-k_{n \perp}^{1}(r)=\mathcal{H}^{2}-\mathcal{H}^{1}+\mathcal{U}^{2} \cos 2 \alpha_{2}-\mathcal{U}^{1} \cos 2 \alpha_{1},
\end{gathered}
$$

we deduce using also the expressions in part a) that

$$
\begin{aligned}
\mathcal{H} & =-\frac{1}{4}\left\{\sin \phi\left[k_{n}^{2}(r)-k_{n}^{1}(r)\right]+\frac{1}{\sin \phi}\left[\kappa\left(r, k_{n \perp}^{2}\right)-\kappa\left(r, k_{n \perp}^{1}\right)\right]\right\} \\
& =\left(\frac{1+\sin ^{2} \phi}{4 \sin ^{2} \phi}\right)\left[\left(\mathcal{H}^{1}-\mathcal{H}^{2}\right)+\left(\frac{\cos ^{2} \phi}{1+\sin ^{2} \phi}\right)\left(\cos \left(2 \alpha_{1}\right) \mathcal{U}^{1}-\cos \left(2 \alpha_{2}\right) \mathcal{U}^{2}\right)\right]
\end{aligned}
$$


COROLlary 3

1) The point $p \in C \backslash M$ is umbilic if and only if $\Upsilon=\Psi=0$, where

$$
\begin{aligned}
\Upsilon & =\left[\tau_{g}^{2}(r)-\tau_{g}^{1}(r)\right]=\mathcal{U}^{2} \sin 2 \alpha_{2}-\mathcal{U}^{1} \sin 2 \alpha_{1}, \\
\Psi & =\sin ^{2} \phi\left[k_{n}^{2}(r)-k_{n}^{1}(r)\right]-\left[k_{n \perp}^{2}(r)-k_{n \perp}^{1}(r)\right] \\
& =\left(\mathcal{H}^{2}-\mathcal{H}^{1}\right) \cos ^{2} \phi+\left(1+\sin ^{2} \phi\right)\left(\mathcal{U}^{1} \cos 2 \alpha_{1}-\mathcal{U}^{2} \cos 2 \alpha_{2}\right) \\
& =\frac{(3-\cos 2 \phi)}{2}\left[\left(\mathcal{H}^{1}-\mathcal{H}^{2}\right) \frac{(1+\cos 2 \phi)}{(3-\cos 2 \phi)}+\left(\mathcal{U}^{1} \cos 2 \alpha_{1}-\mathcal{U}^{2} \cos 2 \alpha_{2}\right)\right] .
\end{aligned}
$$

2) The principal directions at a non-umbilic point $p$ in $C \backslash M$ are characterized by the condition of making an angle $\theta$ with $T_{1}$, given by

$$
\tan 2 \theta=\frac{2\left[\tau_{g}^{2}(r)-\tau_{g}^{1}(r)\right] \sin \phi}{\sin ^{2} \phi\left[k_{n}^{2}(r)-k_{n}^{1}(r)\right]-\left[k_{n \perp}^{2}(r)-k_{n \perp}^{1}(r)\right]}=\frac{2 \Upsilon \sin \phi}{\Psi} .
$$

P r o of. Immediate from Proposition 1.

Proposition 4. Assume that $p=p_{m} \in M$ and that $p_{i}=\Pi_{i}(p)$ are non-umbilic points in $B_{i}$. The normal curvature $k_{n}=k_{n}(\theta)$ of $C$ in the direction of an angle $\theta_{i}$ with the first vector of the parallel basis $\left\{E_{1}^{i}, E_{2}^{i}, N_{i}\right\}$ is given by

$$
k_{n}(\theta)=-\frac{1}{2}\left[\frac{k_{n}^{2}\left(\theta_{2}\right)}{1+r k_{n}^{2}\left(\theta_{2}\right)}-\frac{k_{n}^{1}\left(\theta_{1}\right)}{1+r k_{n}^{1}\left(\theta_{1}\right)}\right] .
$$

Proof. Similar to that of Proposition 1, replacing the frame $\left\{T_{1}, T_{2}, N\right\}$ by any tangent frame to $C$ at $p$.

Remark 2. Taking into account that the two tangent frames differ by an angle $\alpha$, and therefore $\theta_{2}=\theta_{1}+\alpha$ the above equation can be simplified to:

$$
k_{n}(\theta)=-\frac{1}{2}\left[\frac{k_{n}^{2}\left(\theta_{1}+\alpha\right)}{1+r k_{n}^{2}\left(\theta_{1}+\alpha\right)}-\frac{k_{n}^{1}\left(\theta_{1}\right)}{1+r k_{n}^{1}\left(\theta_{1}\right)}\right] .
$$

Remark 3. If both $p_{i}$ are umbilic then the point $p=p_{m}$ is umbilic, the other case leading to umbilic points on $M$ is when the principal frames at $p_{i}$ are parallel and the principal curvatures verify $k_{2}^{2}-k_{2}^{1}=k_{1}^{2}-k_{1}^{1}=-k$. The case $k=0$ is studied in partial detail in next section. Pertinent changes for $k \neq 0$ are made in Remark 4.

3. An introduction to umbilics on conflict surfaces. In this section it will be shown how to determine the Darbouxian type of an umbilic point $p_{m} \in M$. Recall from [1], [4], that this type, $\left(D_{1}, D_{2}, D_{3}\right)$, depends on the 3 -jet of the surface at the point and it determines the behavior of lines of curvature near the umbilic point. To this end in what follows, the 3 -jet of the conflict surface at $p$, in Monge form will be calculated in terms of the corresponding 3 -jets of the surfaces $B_{1}$ and $B_{2}$ at $p_{1}$ and $p_{2}$.

Let the convex surfaces $B_{1}$ and $B_{2}$ be locally given in Monge charts $(x, y)$ and $(u, v)$ by the graphs of

$$
\begin{aligned}
& f_{1}(x, y)=r+\frac{a}{2} x^{2}+\frac{b}{2} y^{2}+\frac{1}{6}\left(a_{30} x^{3}+3 a_{21} x^{2} y+3 a_{12} x y^{2}+a_{30} y^{3}+\ldots\right) \\
& f_{2}(u, v)=-r-\frac{a}{2} u^{2}-\frac{b}{2} v^{2}-\frac{1}{6}\left(b_{30} u^{3}+3 b_{21} u^{2} v+3 b_{12} u v^{2}+b_{30} v^{3}+\ldots\right) .
\end{aligned}
$$


Let $P=(X, Y, Z)$ be a point of $R^{3}$. By the considerations above it follows that $p=(0,0,0) \in C$.

The coordinate expressions of the second order jets at $p$ of the projections $\Pi_{1}(P)$ and $\Pi_{2}(P)$, with targets expressed in the Monge coordinates $(x, y)$ and $(u, v)$ will be computed now.

The projection $\Pi_{1}(X, Y, Z)=(x, y)$, in coordinate expression, is defined implicitly by the equations

$$
\frac{\partial F}{\partial x}=\frac{\partial F}{\partial y}=0,
$$

where $F(x, y, X, Y, Z)=d\left(P, B_{1}\right)^{2}=(X-x)^{2}+(Y-y)^{2}+\left(Z-f_{1}(x, y)\right)^{2}$.

By using the Implicit Function Theorem, after extensive calculation, it is obtained that the solution of the system of equations above is given by

$$
\begin{aligned}
x= & \frac{1}{1+r a} X-\frac{r a_{30}}{2(1+r a)^{3}} X^{2}-\frac{r a_{21}}{(1+r b)(1+r a)^{2}} X Y \\
& \quad-\frac{r a_{12}}{2(1+r a)(1+r b)^{2}} Y^{2}+\frac{a}{(1+r a)^{2}} X Z+\ldots \\
y= & \frac{1}{1+r b} Y-\frac{r a_{21}}{2(1+r b)(1+r a)^{2}} X^{2}-\frac{r a_{12}}{(1+r a)(1+r b)^{2}} X Y \\
& \quad-\frac{r a_{03}}{2(1+r b)^{3}} Y^{2}+\frac{b}{(1+r b)^{2}} Y Z+\ldots
\end{aligned}
$$

Similarly, considering the function

$$
G(x, y, X, Y, Z)=d\left(p, B_{2}\right)^{2}=(X-u)^{2}+(Y-v)^{2}+\left(Z-f_{2}\right)^{2},
$$

we obtain that the projection $\Pi_{2}(X, Y, Z)=(u, v)$, in coordinate expression, is given by

$$
\begin{aligned}
u= & \frac{1}{1+r a} X+\frac{r b_{30}}{2(1+r a)^{3}} X^{2}+\frac{r b_{21}}{(1+r b)(1+r a)^{2}} X Y \\
& +\frac{r b_{12}}{2(1+r a)(1+r b)^{2}} Y^{2}-\frac{a}{(1+r a)^{2}} X Z+\ldots, \\
v= & \frac{1}{1+r b} Y+\frac{r b_{21}}{2(1+r b)(1+r a)^{2}} X^{2}+\frac{r b_{12}}{(1+r a)(1+r b)^{2}} X Y \\
& +\frac{r b_{03}}{2(1+r b)^{3}} Y^{2}-\frac{b}{(1+r b)^{2}} Y Z+\ldots
\end{aligned}
$$

Recall from the Introduction that the conflict surface $C$ is defined by the equation

$$
c(X, Y, Z)=d\left((X, Y, Z),\left(u, v, f_{2}(u, v)\right)\right)-d\left((X, Y, Z),\left(x, y, f_{1}(x, y)\right)\right)=0,
$$

with $(x, y)$ and $(u, v)$ representing the projections $\Pi_{1}(X, Y, Z)$ and $\Pi_{2}(X, Y, Z)$, given by the expressions above.

An extensive calculation leads to the following expression for the 3-jet of the Monge representation of $C$ in a neighborhood of $p=(0,0,0)$ :

$$
\begin{aligned}
Z=\frac{\left(a_{30}-b_{30}\right)}{2(1+a r)^{3}} \frac{X^{3}}{6}+\frac{\left(a_{21}-b_{21}\right)}{2(1+b r)(1+a r)^{2}} \frac{X^{2} Y}{2} \\
\quad+\frac{\left(a_{12}-b_{12}\right)}{2(1+a r)(1+b r)^{2}} \frac{X Y^{2}}{2}+\frac{\left(a_{03}-b_{03}\right)}{2(1+b r)^{3}} \frac{Y^{3}}{6}+\ldots
\end{aligned}
$$


This follows by using the Implicit Function Theorem applied to the function $c(X, Y, Z)$, and observing that $c(0,0,0)=0$ and $\frac{\partial c}{\partial Z}(0,0,0)=4 r \neq 0$.

Since the Darbouxian type of the umbilic is defined by semialgebraic conditions on the coefficients of $j^{3} Z(0,0)$, the behavior of lines of curvature near $p$ can be expressed in terms of the coefficients of the 3 -jets of the surfaces $B_{i}$ at $p_{i}$.

Remark 4. Under generic conditions on the coefficients, the cubic form above determines the index $\left( \pm \frac{1}{2}\right)$ as well as the singularity (hyperbolic, elliptic) and Darbouxian $\left(D_{1}, D_{2}, D_{3}\right)$ types of the umbilic of the conflict surface $C$.

In the example discussed above the caustic umbilic (on the focal surface) is located at infinity. By taking $a+k$ and $b+k, k \neq 0$, instead of $a$ and $b$ in $f_{1}$ and keeping $f_{2}$ unchanged, the caustic umbilic of the conflict surface moves to $\left(0,0, \frac{1}{k}\right)$. The index of the umbilic, as well as the Darbouxian and singularity types of the umbilic will be also determined by the coefficients of the cubic form, changed accordingly to the presence of constant $k$. The generic conditions referred invoked above are expressed by the non-vanishing of a quadratic form, for the index, to which the non-vanishing of pertinent cubic forms should be added for the singularity and Darbouxian classifications [2].

\section{References}

[1] G. Darboux, Sur la forme des lignes de courbure dans le voisinage d'un ombilic, in: Leçons sur la théorie générale des surfaces, Vol. IV, Gauthier-Villars, Paris, 1896, 448-465.

[2] I. R. Porteous, Geometric Differentiation for the Intelligence of Curves and Surfaces, Cambridge Univ. Press, Cambridge, 1994.

[3] D. Siersma, Properties of conflict sets in the plane, this volume.

[4] J. Sotomayor and C. Gutiérrez, Structurally stable configurations of lines of principal curvature, Astérisque 98-99 (1982), 195-215.

[5] J. Sotomayor and C. Gutiérrez, Lines of Curvature and Umbilic Points on Surfaces. Text of Course delivered at the XVIII Brazilian Mathematics Colloquium, IMPA, Rio de Janeiro, 1991.

[6] M. Spivak, A Comprehensive Introduction to Differential Geometry, vols. 1, 3, Publish or Perish, Wilmington, 1979.

[7] J. B. Wilker, Equidistant sets and their connectivity properties, Proc. Amer. Math. Soc. 47 (1975), 446-452. 